INPLASY

PROTOCOL

To cite: Zheng et al. The Efficacy and safety of Sodium Zirconium Cyclosilicate for the treatment of Hyperkalemia: a meta-analysis of randomized controlled trials. Inplasy protocol 202070101. doi: 10.37766/inplasy2020.7.0101

Received: 22 July 2020

Published: 22 July 2020

Corresponding author: Ying Zheng

1127046886@qq.com

Author Affiliation:

Tianjin People's Hospital

Support: None.

Review Stage at time of this submission: The review has not yet started.

Conflicts of interest:

The authors declare no conflict of interest.

\section{The Efficacy and safety of Sodium Zirconium Cyclosilicate for the treatment of Hyperkalemia: a meta-analysis of randomized controlled trials}

\author{
Zheng, Y1; Zhang, H²; Xu, C3; Long, G4.
}

Review question / Objective: P: Patients diagnosed with Hyperkalemia I: The experimental group was treated with Sodium Zirconium Cyclosilicate C: The control group was treated with placebo $O$ : change in serum potassium $S$ : Ramdomized controlled studies.

Condition being studied: Hyperkalemia is the most common electrolyte disturbance. Patients at the highest risk of hyperkalemia are those with chronic kidney disease and heart failure. sodium zirconium cyclosilicate is a highly selective cation exchanger that is not systemically adsorbed, entraps potassium in the gastrointestinal tract in exchange for sodium and hydrogen. But it is unclear the efficacy and safety in patients with hyperkalemia.

INPLASY registration number: This protocol was registered with the International Platform of Registered Systematic Review and Meta-Analysis Protocols (INPLASY) on 22 July 2020 and was last updated on 22 July 2020 (registration number INPLASY202070101).

\section{INTRODUCTION}

Review question / Objective: P: Patients diagnosed with Hyperkalemia I: The experimental group was treated with Sodium Zirconium Cyclosilicate C: The control group was treated with placebo 0 : change in serum potassium $S$ : Ramdomized controlled studies.
Condition being studied: Hyperkalemia is the most common electrolyte disturbance. Patients at the highest risk of hyperkalemia are those with chronic kidney disease and heart failure. sodium zirconium cyclosilicate is a highly selective cation exchanger that is not systemically adsorbed, entraps potassium in the gastrointestinal tract in exchange for 
sodium and hydrogen. But it is unclear the efficacy and safety in patients with hyperkalemia.

\section{METHODS}

Participant or population: Hyperkalemia.

Intervention: Sodium Zirconium Cyclosilicate.

\section{Comparator: Placebo.}

Study designs to be included: Randomized controlled trials.

Eligibility criteria: 1. Patients diagnosed with hyperkalemia 2. Randomized controlled trial 3.availability of full-text publication and there were no language restrictions.

Information sources: We followed PRISMA guidelines to undertake this meta-analysis. We will search PubMed, Embase, Medline, Web of Science, Cochrane Library.The retrieval time was from the time of database establishment to July 2020 .search key words: "Hyperkalemia", "Sodium Zirconium Cyclosilicate".

Main outcome(s): 1. Change in potassium at 48 hours of treatment 2. Change in serum potassium until end of treatment or follow-up during the trial period 3. Adverse effects during the treatment period.

Quality assessment / Risk of bias analysis: Two researchers conducted the quality assessment separately. We will assess the methodological quality of the included studies based on the Cochrane risk of bias tool, including randomized methods, distributive concealment, blinding of participants and personnel, blinding of outcome assessment, incomplete outcome data, selective outcome reporting, and other biases.

Strategy of data synthesis: We will import Literature retrieved in PubMed, Embase, Medline, Web of Science, Cochrane
Library into EndNoteX9.We will design a data extraction table to specify the data scope to be included. The data extraction table contains the following information in each study: author, publication year, country, study design, trial duration, sample size, randomization method, blinding, participants characteristics, intervention measures. The extracted data is summarized in an Excel spreadsheet.

Subgroup analysis: We will conduct subgroup analysis of the included studies according to the study duration, study population.

Sensibility analysis: Sensitivity analysis will be carried out to identify the reliability and stability of aggregation results through eliminating trials with high risk of bias.

\section{Country(ies) involved: China.}

Keywords: Hyperkalemia, sodium zirconium cyclosilicate, Meta-analysis.

Contributions of each author:

Author 1 - Ying Zheng.

Author 2 - Haowen Zhang.

Author 3 - Chengren Xu.

Author 4 - Gang Long. 